\title{
Clathrin locks up vesicle structure
}

\author{
from $H$. Slayter
}

'COATED VESICLES', present in virtually all nucleated cells, were elevated to the status of 'subcellular particle' in 1975, when a method was derived for isolating these rather heterogeneous $(50-150 \mathrm{~nm})$ complex particles. A scarcity of presumed contents following purification notwithstanding, the vesicles appear to be vehicles for the selective transfer of protein molecules into and within cells'. The vesicles are contained in a 'basket' or 'cage' which proves to be formed from polygonal units - pentagons, hexagons and (rarely) heptagons (Fig.1). Particles of various size are coated according to the same plan, in which 12 pentagons are interspersed among a variable number of hexagons.

Purification shows that the basketwork from different sources is remarkably constant, consisting of one major protein, clathrin (MW 180,000), together with small amounts of other proteins of molecular weights 100,000 and 30,000. Recently, several groups have looked at - and/or proteolytically tinkered with - purified clathrin and its penchant for reassembly into empty cages. Ungewickell and Branton $^{2}$ observed reversible dissociation

Fig.1 Large fragments of clathrin cages, negatively stained with uranyl acetate (from Crowther \& Pearse J. Cell Biol. 91, $793 ; 1981)$.

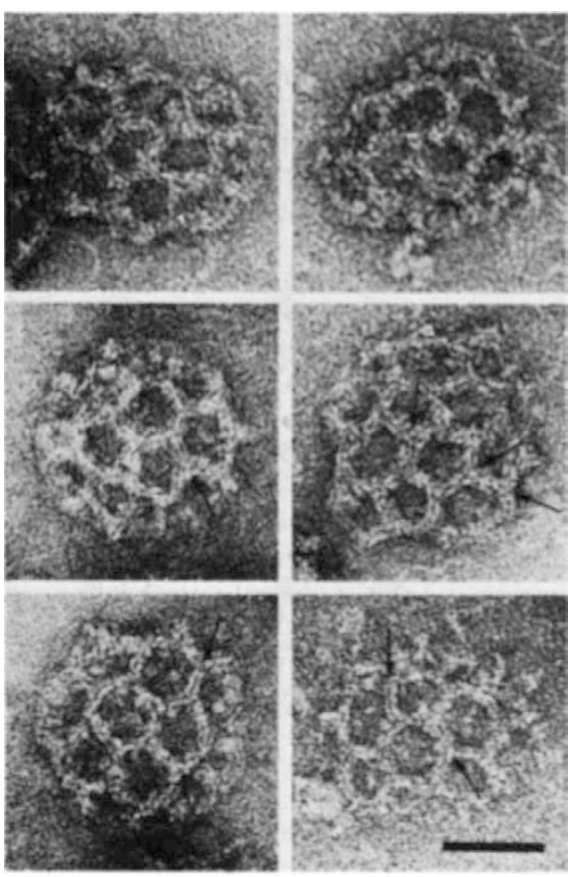

Fig.2 Electron micrographs of $8.4 \mathrm{~S}$ clathrin triskelions (from Ungewickell \& Branton Nature 289, 421; 1981).

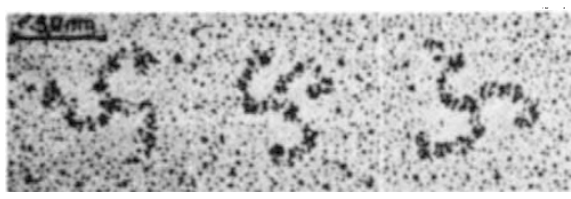

of cages into a unit, consisting of three clathrin monomers and three light chains, which they dubbed 'triskelion' (Fig.2). These $8.4 \mathrm{~S}$ assemblies include three $445 \AA$ legs, each of which is bent in the same direction at a distance of about $190 \AA$ from a common vertex. [Other authors have since reported leg lengths of $433 \AA$ (ref.3) and $504 \AA$ (ref.4) and $160 \AA$ for the proximal segment ${ }^{3}$.] The triskelions are distinctive, not only in morphology but also as a rare but apparently successful example of molecular weight determination by 'drop counting' of electron micrographs after Williams and Backus ${ }^{5}$. The value of 627,000 so obtained is in remarkably close agreement with the 630,000 obtained by sedimentation equilibrium ${ }^{6}$. Unanue, Ungewickell and Branton ${ }^{6}$ have gone on to demonstrate that triskelions bind with high affinity to uncaged vesicles, via protease-sensitive sites. Meanwhile, Kirchausen and Harrison ${ }^{7}$, using soluble homogeneous $8.65 \mathrm{~S}$ clathrin, showed in cross-linking studies that the $8.65 \mathrm{~S}$ material is composed of three $180,000-\mathrm{MW}$ chains and three light chains of MW $33,000-36,000$, and also that the $8.65 \mathrm{~S}$ clathrin can reassemble into cages without the addition of other protein species. The light chain doublet ("clathrin-associated protein' or CAP), which apparently projects from the cage surface ${ }^{8}$, is essential for ptoper cage formation. After de-CAPping by chymotrypsin, pentagons and hexagons still form, but fail to join each other.

A recent extension of these studies shows that elastase digestion ${ }^{4}$, even though it does not shorten the legs, prevents the regular handedness of triskelions. It would seem that the CAP, or light chains, serve to glue the triskelion together somewhere near its vertex. Elastase-treated clathrin associates in extended arrays, rather than sealing itself up into tidy cages. Curiously, however, the $110,000-\mathrm{MW}$ major end product of proteolysed clathrin forms cages (despite visible truncation of the

H. Slayter is in the Sidney Farber Cancer Center, Boston, MA02115. legs), in the absence of light chains, which appear perfectly normal in micrographs.

Crowther and Pearse have applied all the tricks of the trade to obtain the best possible negatively stained electron micrographs of triskelions; that the legs so prepared look 'rather more slender' than those in metal-coated preparations is easily explained in terms of the thickening of the metal coat as well as possible penetration of structures by the negative contract medium. Crowther and Pearse collate various data, proposing a model in which one triskelion is placed at each vertex of the cage (Fig.3). The inner and outer segments of each leg contribute to two successive sides of a pentagon, leaving the last $80 \AA$ of the clathrin monomer unaccounted for. Successive verticles are slightly rotated, producing cross-over packing of the arms. Thus each polygonal side is apparently composed of two proximal legs from adjacent triskelions together with two distal legs from more distant triskelions. The triskelions evidently are flexible enough to associate with equal ease in pentagonal or hexagonal format, and to form surfaces of various curvatures so as to cover different sizes of vesicle. One wonders whether problems in molecular architecture would be more or less complex if all subunits were as accommodating!

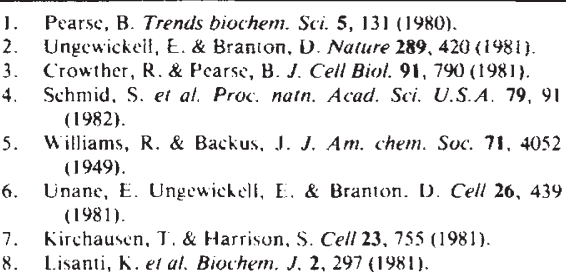

\section{Corrigendum}

In the report from Leg 84 of the Deep Sea Drilling Project, 'Subduction without accretion: Middle America Trench off Guatemala' (Nature 297, 10 June 1982, p.458), we regret that we ommitted the name of one of the authors: Roger Helm, from the Institute of Geology, Rush University, Bochum, FRG. 